\title{
Qi's conjectures on completely monotonic degrees of remainders of asymptotic formulas of di- and trigamma functions
}

\section{Ai-Min $\mathrm{Xu}^{1 *}$ and Zhong-Di Cen ${ }^{1}$}

\section{"Correspondence:}

xuaimin1009@hotmail.com

${ }^{1}$ Institute of Mathematics, Zhejiang

Wanli University, Ningbo, China

\begin{abstract}
Several conjectures posed by Qi on completely monotonic degrees of remainders for the asymptotic formulas of the digamma and trigamma functions are proved.

MSC: 26A48; 33B15; 44A10

Keywords: Complete monotonicity; Completely monotonic degree; Digamma function; Trigamma function; Asymptotic formula
\end{abstract}

\section{Introduction}

A function $f$ is said to be completely monotonic on an interval $I$ if $f$ has derivatives of all orders on $I$ which alternate successively in sign, that is,

$$
(-1)^{n} f^{(n)}(x) \geq 0
$$

for all $x \in I$ and for all $n \geq 0$. See for example [13, Chapterviii], [28, Chap. 1], and [29, Chapter iv]. If inequality (1.1) is strict for all $x \in I$ and for all $n \geq 0$, then $f$ is said to be strictly completely monotonic. Completely monotonic functions have played a very important role in various areas such as the theory of special functions, probability, numerical analysis, and physics.

A notion of completely monotonic degree was invented first in reference [6] and reviewed in the recent paper [22]. It can be used to measure and differentiate complete monotonicity more accurately, and it is also introduced in $[6,8-12,14,16,18-21,25,26]$ and closely related references. Let $f(x)$ be a completely monotonic function on $(0, \infty)$ and denote $f(\infty)=\lim _{x \rightarrow \infty} f(x) \geq 0$. When the function $x^{\alpha}[f(x)-f(\infty)]$ is completely monotonic on $(0, \infty)$ if and only if $0 \leq \alpha \leq r$, the number $r$, denoted by $\operatorname{deg}_{\mathrm{cm}}^{x}[f(x)]$, is called the completely monotonic degree of $f(x)$ with respect to $x \in(0, \infty)$. For more studies on complete monotonicity, the reader is also referred to $[3,8-12,14,16,18-21,25,26]$.

For $x>0$, the classical gamma function

$$
\Gamma(x)=\int_{0}^{\infty} e^{-t} t^{x-1} d t,
$$

(c) The Author(s) 2020. This article is licensed under a Creative Commons Attribution 4.0 International License, which permits use sharing, adaptation, distribution and reproduction in any medium or format, as long as you give appropriate credit to the original author(s) and the source, provide a link to the Creative Commons licence, and indicate if changes were made. The images or other third party material in this article are included in the article's Creative Commons licence, unless indicated otherwise in a credit line to the material. If material is not included in the article's Creative Commons licence and your intended use is not permitted by statutory regulation or exceeds the permitted use, you will need to obtain permission directly from the copyright holder. To view a copy of this licence, visit http://creativecommons.org/licenses/by/4.0/. 
first introduced by the Swiss mathematician Leonhard Euler, is one of the most important functions in mathematical analysis. It often appears in asymptotic series, hypergeometric series, Riemann zeta function, number theory, and so on. It is well known that the digamma function or the psi function is defined by $\psi(x)=\Gamma^{\prime}(x) / \Gamma(x)$, and $\psi^{(i)}(x)(i \geq 1)$ is called the polygamma functions. In particular, $\psi^{\prime}(x)$ is called the trigamma function. In [1], an interesting fact was verified that the function $f(x)=x^{\alpha}[\log (x)-\psi(x)]$ is strictly completely monotonic on $(0, \infty)$ if and only if $\alpha \leq 1$, which improved the results due to Anderson et al. [2]. A new proof was given by [5]. The above result implies that $\operatorname{deg}_{\mathrm{cm}}^{x}[\log (x)-$ $\psi(x)]=1$. It was proved in [27] that the function $x^{2}[\psi(x)-\log (x)]+x / 2$ is strictly decreasing and convex on $(0, \infty)$ and, as $x \rightarrow \infty$, tends to $-1 / 12$. Then the result was improved by [3], and it was proved that the function $\Phi(x)=x^{2}[\psi(x)-\log (x)]+x / 2+1 / 12$ is completely monotonic on $(0, \infty)$. A concise proof of the complete monotonicity of the function $\Phi(x)$ was presented by Qi and Liu [22]. Meanwhile, they proved that

$$
\operatorname{deg}_{\mathrm{cm}}^{x}\left[\Phi(x) / x^{2}\right]=2
$$

Furthermore, Qi and Liu [22] guessed that

$$
\begin{aligned}
& \operatorname{deg}_{\mathrm{cm}}^{x}\left[\log (x)-\frac{1}{2 x}-\psi(x)\right]=1, \\
& \operatorname{deg}_{\mathrm{cm}}^{x}\left[\frac{1}{x}+\frac{1}{2 x^{2}}+\frac{1}{6 x^{3}}-\psi^{\prime}(x)\right]=2, \\
& \operatorname{deg}_{\mathrm{cm}}^{x}\left[\psi^{\prime}(x)-\frac{1}{x}-\frac{1}{2 x^{2}}-\frac{1}{6 x^{3}}+\frac{1}{30 x^{5}}\right]=4 .
\end{aligned}
$$

Equations (1.3), (1.2), (1.4), and (1.5) can be written concisely as follows:

$$
\begin{aligned}
& \operatorname{deg}_{\mathrm{cm}}^{x}\left[(-1) R_{0}^{\prime}(x)\right]=1, \\
& \operatorname{deg}_{\mathrm{cm}}^{x}\left[(-1) R_{1}^{\prime}(x)\right]=2, \\
& \operatorname{deg}_{\mathrm{cm}}^{x}\left[(-1)^{2} R_{1}^{\prime \prime}(x)\right]=2, \\
& \operatorname{deg}_{\mathrm{cm}}^{x}\left[(-1)^{2} R_{2}^{\prime \prime}(x)\right]=4,
\end{aligned}
$$

if we let

$$
\begin{aligned}
R_{n}(x)= & (-1)^{n}\left[\log \Gamma(x)-\left(x-\frac{1}{2}\right) \log (x)+x-\frac{1}{2} \log (2 \pi)\right. \\
& -\sum_{k=1}^{n} \frac{B_{2 k}}{(2 k-1) 2 k} \frac{1}{\left.x^{2 k-1}\right]}
\end{aligned}
$$

where $B_{i}$ denotes the $i$ th Bernoulli number defined by the generating function

$$
\frac{t}{e^{t}-1}=\sum_{i=1}^{\infty} \frac{B_{i}}{i !} t^{i} .
$$

In fact, $R_{n}(x)(n \geq 0)$ was proved to be completely monotonic on $(0, \infty)$ in [1] and [7]. In [11], the completely monotonic degree of the function $R_{n}(x)$ for $n \geq 0$ on $(0, \infty)$ was 
proved to be at least $n$. This means that the functions $(-1)^{m}\left[R_{n}(x)\right]^{(m)}$ for $m, n \geq 0$ are completely monotonic on $(0, \infty)$. Qi and Liu [22] asked a question: What are the completely monotonic degrees of the completely monotonic functions $(-1)^{m}\left[R_{n}(x)\right]^{(m)}$ for $m, n \geq 0$ on $(0, \infty)$ ? Several conjectures posed by Qi can be recited as follows:

(1) The completely monotonic degrees of $R_{n}(x)$ for $n \geq 0$ with respect to $x \in(0, \infty)$ satisfy

$$
\operatorname{deg}_{\mathrm{cm}}^{x}\left[R_{0}(x)\right]=0, \quad \operatorname{deg}_{\mathrm{cm}}^{x}\left[R_{1}(x)\right]=1,
$$

and

$$
\operatorname{deg}_{\mathrm{cm}}^{x}\left[R_{n}(x)\right]=2(n-1), \quad n \geq 2 ;
$$

(2) The completely monotonic degrees of $-R_{n}^{\prime}(x)$ for $n \geq 0$ with respect to $x \in(0, \infty)$ satisfy

$$
\operatorname{deg}_{\mathrm{cm}}^{x}\left[-R_{0}^{\prime}(x)\right]=1, \quad \operatorname{deg}_{\mathrm{cm}}^{x}\left[-R_{1}^{\prime}(x)\right]=2,
$$

and

$$
\operatorname{deg}_{\mathrm{cm}}^{x}\left[-R_{n}^{\prime}(x)\right]=2 n-1, \quad n \geq 2 ;
$$

(3) The completely monotonic degrees of $(-1)^{m} R_{n}^{(m)}(x)$ for $m \geq 2$ and $n \geq 0$ with respect to $x \in(0, \infty)$ satisfy

$$
\operatorname{deg}_{\mathrm{cm}}^{x}\left[(-1)^{m}\left[R_{0}(x)\right]^{(m)}\right]=m-1, \quad \operatorname{deg}_{\mathrm{cm}}^{x}\left[(-1)^{m}\left[R_{1}(x)\right]^{(m)}\right]=m,
$$

and

$$
\operatorname{deg}_{\mathrm{cm}}^{x}\left[(-1)^{m}\left[R_{n}(x)\right]^{(m)}\right]=m+2(n-1), \quad n \geq 2 .
$$

In this paper, following the method due to Koumandos and Pedersen [11], we prove that

$$
\begin{aligned}
& \operatorname{deg}_{\mathrm{cm}}^{x}\left[(-1)^{2} R_{0}^{\prime \prime}(x)\right]=2, \\
& \operatorname{deg}_{\mathrm{cm}}^{x}\left[(-1)^{2} R_{1}^{\prime \prime}(x)\right]=3, \\
& \operatorname{deg}_{\mathrm{cm}}^{x}\left[-R_{0}^{\prime}(x)\right]=1,
\end{aligned}
$$

and

$$
\operatorname{deg}_{\mathrm{cm}}^{x}\left[-R_{1}^{\prime}(x)\right]=2
$$

Eqs. (1.7) and (1.8) show that it may be not true that $\operatorname{deg}_{\mathrm{cm}}^{x}\left[(-1)^{m}\left[R_{0}(x)\right]^{(m)}\right]=m-1$ and $\operatorname{deg}_{\mathrm{cm}}^{x}\left[(-1)^{m}\left[R_{1}(x)\right]^{(m)}\right]=m$ for all $m \geq 2$.

It is worth noting that almost at the same time that we submitted our paper, Qi and Mahmoud [23, 24] independently confirmed (1.9) and (1.10). Meanwhile, motivated by 
(1.7) and (1.8), which can also be verified as in [15], Qi and Mahmoud [23, 24] corrected and modified two conjectures stated in (3) as follows: The completely monotonic degrees of $(-1)^{m} R_{n}^{(m)}(x)$ for $m \geq 2$ with respect to $x \in(0, \infty)$ satisfy

$$
\operatorname{deg}_{\mathrm{cm}}^{x}\left[(-1)^{m}\left[R_{0}(x)\right]^{(m)}\right]=m, \quad \operatorname{deg}_{\mathrm{cm}}^{x}\left[(-1)^{m}\left[R_{1}(x)\right]^{(m)}\right]=m+1 .
$$

\section{A lemma}

A necessary and sufficient condition for complete monotonicity was given by Bernstein's theorem (see Theorem $12 \mathrm{~b}$ in $[29, \mathrm{p} .161]$ ), which states that $f$ is completely monotonic on $(0, \infty)$ if and only if

$$
f(x)=\int_{0}^{\infty} e^{-x t} d \mu(t)
$$

where $\mu$ is nondecreasing and the integral converges for $0<x<\infty$. Bernstein's theorem was extended by Koumandos and Pedersen [11]. The theorem of Koumandos and Pedersen is a key and useful tool in our paper. Now, we state it as a lemma.

Lemma 1 ([11], Theorem 1.3) Let $r \geq 1$ be an integer. The function $x^{r} f(x)$ is completely monotonic if and only if

$$
f(x)=\int_{0}^{\infty} p(t) e^{-x t} d t
$$

where the integral converges for all $x>0$ and $p$ is $r-1$ times differentiable on $[0, \infty)$ with $p^{(r-1)}(t)=\mu([0, t])$ for some Radon measure $\mu$ and $p^{(k)}(0)=0$ for $0 \leq k \leq r-2$.

The theorem of Koumandos and Pedersen contains a simple characterization of completely monotonic functions like $x^{r} f(x)$. It is very helpful for us to evaluate the completely monotonic degrees for certain functions.

\section{Proofs of the conjectures}

Now, we present our theorems and proofs.

Theorem 1 For $x>0$, we have

$$
\operatorname{deg}_{\mathrm{cm}}^{x}\left[(-1)^{2} R_{0}^{\prime \prime}(x)\right]=2 .
$$

Proof Let $\phi(x)=(-1)^{2} R_{0}^{\prime \prime}(x)=\psi^{\prime}(x)-\frac{1}{x}-\frac{1}{2 x^{2}}$. It follows from Theorem 9 in [1] that $\lim _{x \rightarrow \infty} \phi(x)=0$. Using Binet's formula [4, p. 8], we obtain the representation

$$
\phi(x)=\int_{0}^{\infty}\left(\frac{t}{e^{t}-1}+\frac{t}{2}-1\right) e^{-x t} d t=\int_{0}^{\infty} p(t) e^{-x t} d t,
$$

where

$$
p(t)= \begin{cases}\frac{t}{e^{t}-1}+\frac{t}{2}-1, & t>0, \\ 0, & t=0 .\end{cases}
$$


It is obvious that $p(t)$ and $p^{\prime}(t)$ are right-continuous at $t=0$ and the right-derivative $p_{+}^{\prime}(0)=$ 0 . For $t>0$, we have

$$
p^{\prime \prime}(t)=\frac{e^{t}}{\left(e^{t}-1\right)^{3}}\left[(t-2) e^{t}+t+2\right]
$$

Write $g(t)=(t-2) e^{t}+t+2$. We find $g(0)=0, g^{\prime}(0)=0$, and $g^{\prime \prime}(t)=t e^{t}>0$ for $t>0$. Thus, we have $g^{\prime}(t)>0$ and $g(t)>0$ for $t>0$, which implies $p^{\prime \prime}(t)>0$ for $t>0$. So we have that $p^{\prime}(t)$ is a Radon measure on $[0, \infty)$. It follows from Lemma 1 that $x^{2} \phi(x)$ is completely monotonic on $(0, \infty)$, which implies that

$$
\operatorname{deg}_{\mathrm{cm}}^{x}\left[(-1)^{2} R_{0}^{\prime \prime}(x)\right] \geq 2
$$

Next, we assume that $x^{\alpha} \phi(x)$ is completely monotonic on $(0, \infty)$. Then, for all $x>0$, we have

$$
\left[x^{\alpha} \phi(x)\right]^{\prime}=\alpha x^{\alpha-1} \phi(x)+x^{\alpha} \phi^{\prime}(x) \leq 0
$$

which implies

$$
\alpha \leq-\frac{x \phi^{\prime}(x)}{\phi(x)}=-\frac{x\left(\psi^{\prime \prime}(x)+\frac{1}{x^{2}}+\frac{1}{x^{3}}\right)}{\psi^{\prime}(x)-\frac{1}{x}-\frac{1}{2 x^{2}}}=-\frac{x^{3} \psi^{\prime \prime}(x)+x+1}{x^{2} \psi^{\prime}(x)-x-\frac{1}{2}} .
$$

Since $\lim _{x \rightarrow 0} x^{2} \psi^{\prime}(x)=1$ and $\lim _{x \rightarrow 0} x^{3} \psi^{\prime \prime}(x)=-2$, we get $\alpha \leq 2$ if we let $x$ tend to 0 . Thus, we have

$$
\operatorname{deg}_{\mathrm{cm}}^{x}\left[(-1)^{2} R_{0}^{\prime \prime}(x)\right] \leq 2
$$

From (3.2) and (3.3) we have (3.1). Then the proof is complete.

Theorem 2 For $x>0$, we have

$$
\operatorname{deg}_{\mathrm{cm}}^{x}\left[(-1)^{2} R_{1}^{\prime \prime}(x)\right]=3
$$

Proof Let $\phi(x)=(-1)^{2} R_{1}^{\prime \prime}(x)=\frac{1}{x}+\frac{1}{2 x^{2}}+\frac{1}{6 x^{3}}-\psi^{\prime}(x)$. From Theorem 9 in [1] we obtain $\lim _{x \rightarrow \infty} \phi(x)=0$. By Binet's formula [4, p. 8], we obtain the representation

$$
\phi(x)=\int_{0}^{\infty}\left(-\frac{t}{e^{t}-1}-\frac{t}{2}+\frac{t^{2}}{12}+1\right) e^{-x t} d t=\int_{0}^{\infty} p(t) e^{-x t} d t
$$

where

$$
p(t)= \begin{cases}-\frac{t}{e^{t}-1}-\frac{t}{2}+\frac{t^{2}}{12}+1, & t>0 \\ 0, & t=0 .\end{cases}
$$

It is easy to check that $p(t), p^{\prime}(t)$, and $p^{\prime \prime}(t)$ are right-continuous at $t=0$, and the rightderivatives $p_{+}^{\prime}(0)=p_{+}^{\prime \prime}(0)=0$. For $t>0$, we have

$$
p^{\prime \prime \prime}(t)=\frac{e^{t}}{\left(e^{t}-1\right)^{4}}\left[(t-3) e^{2 t}+4 t e^{t}+t+3\right] \text {. }
$$


Write $g(t)=(t-3) e^{2 t}+4 t e^{t}+t+3$. We find $g^{(k)}(0)=0$ for $k=0,1, \ldots, 4, g^{(5)}(0)=4$, and $g^{(6)}(t)=64 t e^{2 t}+4(t+6) e^{t}$. Thus, we have $g^{(k)}(t)>0$ for $t>0$ and $k=0,1, \ldots, 6$, which implies $p^{\prime \prime \prime}(t)>0$ for $t>0$. So we have that $p^{\prime \prime}(t)$ is a Radon measure on $[0, \infty)$. It follows from Lemma 1 that $x^{3} \phi(x)$ is completely monotonic on $(0, \infty)$, which implies that

$$
\operatorname{deg}_{\mathrm{cm}}^{x}\left[(-1)^{2} R_{1}^{\prime \prime}(x)\right] \geq 3
$$

Next, we assume that $x^{\alpha} \phi(x)$ is completely monotonic on $(0, \infty)$. Then, for all $x>0$, we have

$$
\left[x^{\alpha} \phi(x)\right]^{\prime}=\alpha x^{\alpha-1} \phi(x)+x^{\alpha} \phi^{\prime}(x) \leq 0,
$$

which implies

$$
\alpha \leq-\frac{x \phi^{\prime}(x)}{\phi(x)}=-\frac{x\left(-\frac{1}{x^{2}}-\frac{1}{x^{3}}-\frac{1}{2 x^{4}}-\psi^{\prime \prime}(x)\right)}{\frac{1}{x}+\frac{1}{2 x^{2}}+\frac{1}{6 x^{3}}-\psi^{\prime}(x)}=\frac{x^{4} \psi^{\prime \prime}(x)+x^{2}+x+\frac{1}{2}}{x^{2}+\frac{1}{2} x+\frac{1}{6}-x^{3} \psi^{\prime}(x)} .
$$

Since $\lim _{x \rightarrow 0} x^{2} \psi^{\prime}(x)=1$ and $\lim _{x \rightarrow 0} x^{3} \psi^{\prime \prime}(x)=-2$, we get $\alpha \leq 3$ if we let $x$ tend to 0 . Thus, we have

$$
\operatorname{deg}_{\mathrm{cm}}^{x}\left[(-1)^{2} R_{1}^{\prime \prime}(x)\right] \leq 3
$$

From (3.5) and (3.6) we conclude that (3.4) is true. Then the proof is complete.

Remark 1 It follows from the conjectures of Qi that $\operatorname{deg}_{\mathrm{cm}}^{x}\left[(-1)^{2} R_{0}^{\prime \prime}(x)\right]=1$ and $\operatorname{deg}_{\mathrm{cm}}^{x}\left[(-1)^{2} R_{1}^{\prime \prime}(x)\right]=2$. According to Theorems 1 and 2 , we correct them and get $\operatorname{deg}_{\mathrm{cm}}^{x}\left[(-1)^{2} R_{0}^{\prime \prime}(x)\right]=2$ and $\operatorname{deg}_{\mathrm{cm}}^{x}\left[(-1)^{2} R_{1}^{\prime \prime}(x)\right]=3$.

Theorem 3 For $x>0$, we have

$$
\operatorname{deg}_{\mathrm{cm}}^{x}\left[-R_{0}^{\prime}(x)\right]=1
$$

Proof Let $\phi(x)=-R_{0}^{\prime}(x)=\log (x)-\frac{1}{2 x}-\psi(x)$. It is known that $\lim _{x \rightarrow \infty} \phi(x)=0$. Using Binet's formula $[4$, p. 8$]$, we obtain the representation

$$
\begin{aligned}
\phi(x) & =\int_{0}^{\infty}\left(\frac{1}{1-e^{-t}}-\frac{1}{t}-\frac{1}{2}\right) e^{-x t} d t=\int_{0}^{\infty}\left(\frac{1}{e^{t}-1}-\frac{1}{t}+\frac{1}{2}\right) e^{-x t} d t \\
& =\int_{0}^{\infty} p(t) e^{-x t} d t,
\end{aligned}
$$

where

$$
p(t)= \begin{cases}\frac{1}{e^{t}-1}-\frac{1}{t}+\frac{1}{2}, & t>0 \\ 0, & t=0\end{cases}
$$

It is easy to check $p(t)$ is right-continuous at $t=0$, and for $t>0$,

$$
p^{\prime}(t)=-\frac{1}{e^{t}-1}-\frac{1}{\left(e^{t}-1\right)^{2}}+\frac{1}{t^{2}}
$$


Since $e^{t}>1+t+\frac{t^{2}}{2}+\frac{t^{3}}{6}$, we have

$$
p^{\prime}(t)>-\frac{1}{t+\frac{t^{2}}{2}+\frac{t^{3}}{6}}-\frac{1}{\left(t+\frac{t^{2}}{2}+\frac{t^{3}}{6}\right)^{2}}+\frac{1}{t^{2}}=\frac{t^{2}+3}{\left(t^{2}+3 t+6\right)^{2}}>0 .
$$

So we have that $p(t)$ is a Radon measure on $[0, \infty)$. It follows from Lemma 1 that $x \phi(x)$ is completely monotonic on $(0, \infty)$, which implies that

$$
\operatorname{deg}_{\mathrm{cm}}^{x}\left[-R_{0}^{\prime}(x)\right] \geq 1
$$

On the other hand, we assume that $x^{\alpha} \phi(x)$ is completely monotonic on $(0, \infty)$. Then, for all $x>0$, we have

$$
\left(x^{\alpha} \phi(x)\right)^{\prime}=\alpha x^{\alpha-1} \phi(x)+x^{\alpha} \phi^{\prime}(x) \leq 0
$$

which implies

$$
\alpha \leq-\frac{x \phi^{\prime}(x)}{\phi(x)}=-\frac{x\left(\frac{1}{x}+\frac{1}{2 x^{2}}-\psi^{\prime}(x)\right)}{\log (x)-\frac{1}{2 x}-\psi(x)}=-\frac{x+\frac{1}{2}-x^{2} \psi^{\prime}(x)}{x(\log (x)-\psi(x))-\frac{1}{2}} .
$$

Since $\lim _{x \rightarrow 0} x[\log (x)-\psi(x)]=1$ (see [2]) and $\lim _{x \rightarrow 0} x^{2} \psi^{\prime}(x)=1$, we get $\alpha \leq 1$ if we let $x$ tend to 0 . Thus, we have

$$
\operatorname{deg}_{\mathrm{cm}}^{x}\left[-R_{0}^{\prime}(x)\right] \leq 1
$$

From (3.8) and (3.9) we conclude that (3.7) is true. Then the proof is complete.

Remark 2 In fact, in reference [5] and closely related references therein, it was proved that the function $x[\log (x)-\psi(x)]$ is completely monotonic on $(0, \infty)$ and that

$$
\lim _{x \rightarrow \infty}\{x[\log (x)-\psi(x)]\}=\frac{1}{2}
$$

This implies that the function

$$
x[\log (x)-\psi(x)]-\frac{1}{2}=x\left\{[\log (x)-\psi(x)]-\frac{1}{2 x}\right\}
$$

is also completely monotonic on $(0, \infty)$ and that it tends to 0 as $x \rightarrow \infty$. Hence, the function $[\log (x)-\psi(x)]-1 /(2 x)$ is completely monotonic and its completely monotonic degree is 1 at least. This result can be applied to prove the above theorem.

Theorem 4 For $x>0$, we have

$$
\operatorname{deg}_{\mathrm{cm}}^{x}\left[-R_{1}^{\prime}(x)\right]=2 .
$$

Proof Let $\phi(x)=-R_{1}^{\prime}(x)=\psi(x)-\log (x)+\frac{1}{2 x}+\frac{1}{12 x^{2}}$, and we have $\lim _{x \rightarrow \infty} \phi(x)=0$. It follows from (14) in [17] that

$$
\phi(x)=\int_{0}^{\infty}\left(-\frac{1}{e^{t}-1}+\frac{1}{t}+\frac{t}{12}-\frac{1}{2}\right) e^{-x t} d t=\int_{0}^{\infty} p(t) e^{-x t} d t
$$


where

$$
p(t)= \begin{cases}-\frac{1}{e^{t}-1}+\frac{1}{t}+\frac{t}{12}-\frac{1}{2}, & t>0, \\ 0, & t=0 .\end{cases}
$$

It is easy to check that $p(t)$ and $p^{\prime}(t)$ are right-continuous at $t=0$ and the right-derivative of $p(t)$ at $t=0$ is zero, that is, $p_{+}^{\prime}(0)=0$. For $t>0$, we have

$$
\begin{aligned}
p^{\prime \prime}(t)= & \frac{2}{t^{3}}-\frac{1}{e^{t}-1}-\frac{3}{\left(e^{t}-1\right)^{2}}-\frac{2}{\left(e^{t}-1\right)^{3}} \\
> & \frac{2}{t^{3}}-\frac{1}{t+\frac{t^{2}}{2}+\frac{t^{3}}{6}+\frac{t^{4}}{24}+\frac{t^{5}}{120}}-\frac{3}{\left(t+\frac{t^{2}}{2}+\frac{t^{3}}{6}+\frac{t^{4}}{24}+\frac{t^{5}}{120}\right)^{2}} \\
& -\frac{2}{\left(t+\frac{t^{2}}{2}+\frac{t^{3}}{6}+\frac{t^{4}}{24}+\frac{t^{5}}{120}\right)^{3}} \\
= & \frac{2 t\left(t^{8}+15 t^{4}+75 t^{6}+305 t^{5}+960 t^{4}+2100 t^{3}+3800 t^{2}+3600 t+7200\right)}{\left(t^{4}+5 t^{3}+20 t^{2}+60 t+120\right)^{3}}
\end{aligned}
$$

$>0$.

So we have that $p^{\prime}(t)$ is a Radon measure on $[0, \infty)$. According to Lemma 1 , we conclude that $x^{2} \phi(x)$ is completely monotonic on $(0, \infty)$, which implies that

$$
\operatorname{deg}_{\mathrm{cm}}^{x}\left[-R_{1}^{\prime}(x)\right] \geq 2
$$

For the proof of $\operatorname{deg}_{\mathrm{cm}}^{x}\left[-R_{1}^{\prime}(x)\right] \leq 2$, see [22].

Remark 3 In reference [3], it was proved that the function

$$
\Phi(x)=x^{2}[\psi(x)-\log (x)]+\frac{x}{2}+\frac{1}{12}=x^{2}\left[\psi(x)-\log (x)+\frac{1}{2 x}+\frac{1}{12 x^{2}}\right]
$$

is completely monotonic on $(0, \infty)$ and that it tends to 0 as $x \rightarrow \infty$. This implies that the completely monotonic degree of the function $\psi(x)-\log (x)+\frac{1}{2 x}+\frac{1}{12 x^{2}}$ is 2 at least. This is also a part of the proof of Theorem 2 in reference [22] by Qi and Liu. Our method, with the help of the Koumandos-Pedersen theorem, is the same as that of Qi and Liu, a method of integration-by-part, essentially.

Remark 4 Almost at the same time that we submitted our paper, Qi and Mahmoud [23, 24] independently proved Theorems 3 and 4 .

Remark 5 In the proof of Theorems 1 and 4, Koumandos and Pedersen's theorem is applied with $r=2$, in the proof of Theorem 2 with $r=3$, and in the proof of Theorem 3 with $r=1$. However, Koumandos and Pedersen's theorem cannot be applied with an $r$ one unity larger in Theorems 1, 2, 3, and 4. Let us take the proof of Theorem 1 as an example. In the proof of Theorem 1, we got

$$
p^{\prime \prime}(t)=\frac{e^{t}}{\left(e^{t}-1\right)^{3}}\left[(t-2) e^{t}+t+2\right]
$$


and proved $p^{\prime \prime}(t)>0$ for $t>0$. So we obtained $\operatorname{deg}_{\mathrm{cm}}^{x}\left[(-1)^{2} R_{0}^{\prime \prime}(x)\right] \geq 2$. In fact, we can further obtain

$$
p^{\prime \prime \prime}(t)=-\frac{e^{t}}{\left(e^{t}-1\right)^{4}}\left[(t-3) e^{2 t}+4 t e^{t}+t+3\right] .
$$

According to the proof of Theorem 2, we can prove $p^{\prime \prime \prime}(t)<0$ for $t>0$ and get that $p^{\prime \prime}(t)$ is not increasing on $[0, \infty)$. Thus, the function $p^{\prime \prime}(t)$ is not a Radon measure on $[0, \infty)$. It means that the function $p(t)$ in Theorem 1 does not satisfy the conditions of Lemma 1 with $r=3$. In other words, we cannot get that $x^{3} R_{0}^{\prime \prime}(x)$ is completely monotonic on $(0, \infty)$ and $\operatorname{deg}_{\mathrm{cm}}^{x}\left[(-1)^{2} R_{0}^{\prime \prime}(x)\right] \geq 3$. Similar discussion can be carried out in the proofs of the other three theorems.

\section{Acknowledgements}

We are very grateful to the anonymous referees for their very helpful and detailed comments.

\section{Funding}

Ai-Min Xu's work was supported by the Natural Science Foundation of Zhejiang Province (Grant No. LY18A010001) and the Ningbo Natural Science Foundation (Grant No. 2019A610038). Zhong-Di Cen's work was supported by Zhejiang Province Public Welfare Technology Application Research Project (Grant No. LGF19A010001) and the Ningbo Natural Science Foundation (Grant No. 2019A610045).

\section{Availability of data and materials}

Not applicable.

\section{Competing interests}

The authors declare that they have no competing interests.

\section{Authors' contributions}

All authors contributed equally to the writing of this paper. All authors read and approved the final manuscript.

\section{Publisher's Note}

Springer Nature remains neutral with regard to jurisdictional claims in published maps and institutional affiliations.

Received: 28 November 2019 Accepted: 18 March 2020 Published online: 30 March 2020

\section{References}

1. Alzer, H.: On some inequalities for the gamma and psi functions. Math. Compet. 66, 373-389 (1997). https://doi.org/10.2307/2153660

2. Anderson, G.D., Barnard, R.W., Richards, K.C., Vamanamurthy, M.K., Vuorinen, M.: Inequalities for zero-balanced hypergeometric functions. Trans. Am. Math. Soc. 347, 1713-1723 (1995). https://doi.org/10.2307/2154966

3. Chen, C.-P., Qi, F., Srivastava, H.M.: Some properties of functions related to the gamma and psi functions. Integral Transforms Spec. Funct. 21, 153-164 (2010). https://doi.org/10.1016/j.camwa.2011.08.053

4. Erdélyi, A.: Higher Transcendental Functions, vol. 1. McGraw-Hill, New York (1953)

5. Guo, B.-N., Qi, F.: Two new proofs of the complete monotonicity of a function involving the psi function. Bull. Korean Math. Soc. 47, 103-111 (2010). https://doi.org/10.4134/BKMS.2010.47.1.103

6. Guo, B.-N., Qi, F.: A completely monotonic function involving the tri-gamma function and with degree one. Appl. Math. Comput. 218, 9890-9897 (2012). https://doi.org/10.1016/j.amc.2012.03.075

7. Koumandos, S.: Remarks on some completely monotonic functions. J. Math. Anal. Appl. 324, 1458-1461 (2006) https://doi.org/10.1016/j.jmaa.2005.12.017

8. Koumandos, S.: Monotonicity of some functions involving the gamma and psi functions. Math. Compet. 77, 2261-2275 (2008). https://doi.org/10.1090/s0025-5718-08-02140-6

9. Koumandos, S., Lamprecht, M.: Some completely monotonic functions of positive order. Math. Compet. 79, 1697-1707 (2010). https://doi.org/10.1090/s0025-5718-09-02313-8

10. Koumandos, S., Lamprecht, M.: Complete monotonicity and related properties of some special functions. Math. Compet. 82, 1097-1120 (2013). https://doi.org/10.1090/s0025-5718-2012-02629-9

11. Koumandos, S., Pedersen, H.L.: Completely monotonic functions of positive order and asymptotic expansions of the logarithm of Barnes double gamma function and Euler's gamma function. J. Math. Anal. Appl. 355, 33-40 (2009). https://doi.org/10.1016/j.jmaa.2009.01.042

12. Koumandos, S., Pedersen, H.L.: Absolutely monotonic functions related to Euler's gamma function and Barnes' double and triple gamma function. Monatshefte Math. 163, 51-69 (2011). https://doi.org/10.1007/s00605-010-0197-9

13. Mitrinović, D.S., Pečarić, J.E., Fink, A.M.: Classical and New Inequalities in Analysis. Kluwer Academic, Dordrecht (1993)

14. Qi, F.: Properties of modified Bessel functions and completely monotonic degrees of differences between exponential and trigamma functions. Math. Inequal. Appl. 18, 493-518 (2015). https://doi.org/10.7153/mia-18-37 
15. Qi, F.: A double inequality for the ratio of two non-zero neighbouring Bernoulli numbers. J. Comput. Appl. Math. 351, 1-5 (2019). https://doi.org/10.1016/j.cam.2018.10.049

16. Qi, F., Agarwal, R.P.: On complete monotonicity for several classes of functions related to ratios of gamma functions J. Inequal. Appl. 2019, 36 (2019). https://doi.org/10.1186/s13660-019-1976-z

17. Qi, F., Cui, R.-Q., Chen, C.-P., Guo, B.-N.: Some completely monotonic functions involving polygamma functions and an application. J. Math. Anal. Appl. 310, 303-308 (2005). https://doi.org/10.1016/j.jmaa.2005.02.016

18. Qi, F., Guo, B.-N.: Lévy-Khintchine representation of Toader-Qi mean. Math. Inequal. Appl. 21, 421-431 (2018). https://doi.org/10.7153/mia-2018-21-29

19. Qi, F., Guo, B.-N.: The reciprocal of the weighted geometric mean of many positive numbers is a Stieltjes function. Quaest. Math. 41,653-664 (2018). https://doi.org/10.13140/RG.2.2.23822.36163

20. Qi, F., Li, W.-H.: Integral representations and properties of some functions involving the logarithmic function. Filomat 30, 1659-1674 (2016). https://doi.org/10.2298/FIL1607659Q

21. Qi, F., Lim, D.: Integral representations of bivariate complex geometric mean and their applications. J. Comput. Appl. Math. 330, 41-58 (2018). https://doi.org/10.1016/j.cam.2017.11.047

22. Qi, F., Liu, A.-Q.: Completely monotonic degrees for a difference between the logarithmic and psi functions. J. Comput. Appl. Math. 361, 366-371 (2019). https://doi.org/10.1016/j.cam.2019.05.001

23. Qi, F., Mahmoud, M.: Completely monotonic degrees of remainders of asymptotic expansions of the digamma function (2019). arXiv:1912.07989

24. Qi, F., Mahmoud, M.: Completely monotonic degrees of remainders of asymptotic expansions of the digamma function (2019). HAL preprint, available online at https://hal.archives-ouvertes.fr/hal-02415224

25. Qi, F., Zhang, X.-.., Li, W.-H.: Lévy-Khintchine representation of the weighted geometric mean and the logarithmic mean. Mediterr. J. Math. 11, 315-327 (2014). https://doi.org/10.1007/s00009-013-0311-z

26. Qi, F., Zhang, X.-J., Li, W.-H.: The harmonic and geometric means are Bernstein function. Bol. Soc. Mat. Mex. 23, 713-736 (2017). https://doi.org/10.1007/s40590-016-0085-y

27. Qiu, S.-L., Vuorinen, M.: Some properties of the gamma and psi functions, with applications. Math. Compet. 75, 723-742 (2005). https://doi.org/10.1090/S0025-5718-04-01675-8

28. Schilling, R.L., Song, R., Vondraček, Z.: Bernstein Functions-Theory and Applications, 2nd edn. de Gruyter Studies in Mathematics, vol. 37. de Gruyter, Berlin (2012)

29. Widder, D.V:: The Laplace Transform. Princeton University Press, Princeton (1946)

\section{Submit your manuscript to a SpringerOpen ${ }^{\circ}$ journal and benefit from:}

- Convenient online submission

- Rigorous peer review

- Open access: articles freely available online

- High visibility within the field

- Retaining the copyright to your article

Submit your next manuscript at $\boldsymbol{~ s p r i n g e r o p e n . c o m ~}$ 